\title{
MANEUVERING TARGET MOTION ANALYSIS USING HIDDEN MARKOV MODEL.'
}

\author{
Olivier TRÉMOIS, J.P. LE CADRE.
}

\author{
IRISA/CNRS, Campus de Beaulieu, 35042 RENNES CEDEX
}

\begin{abstract}
The basic problem of target motion analysis (TMA) is to estimate the trajectory of an object (i.e. position and velocity for a rectilinear movement) from noise corrupted sensor data. The problem is quite easy for a rectilinear movement of the source, but numerous problem arise when it maneuvers. Our main goal is to apply hidden markov modelling to this, and to optimize the estimability of the source trajectory via the observer motion.
\end{abstract}

\section{INTRODUCTION.}

The basic problem of target motion analysis (TMA) is to estimate the trajectory of an object (i.e. position and velocity for a rectilinear movement) from noise corrupted sensor data. The problem is not new and numerous approaches of the maneuvering target problem have appeared. Most of them use a prior information on the maneuver they want to detect. In general they use different kinematic models of the source's motion and they switch from one to the other according to a likelihood ratio. These models are quite efficient when the data have the right statistical properties, but their performances decrease dramatically when they try to process data coming from real scenarios.

Recently solutions using hidden Markov model (HMM) appeared. The basic idea is to dicretize the state space (position and velocity) and to provide a Markov model of the source's motion. This type of model is an elegant solution to the maneuvering target tracking problem because it does not requires any prior information on the maneuvers, so that its performance does not depend on some acute criterion which hardly occurs in real scenarios.

Our contribution to this work is to apply HMM to bearings only target motion analysis, and to analyse the statistical performances of this type of model in these conditions. After that one can optimize the estimation of the trajectory of the target, by controlling the Markov chain via the observer motion.

\section{BEARINGS ONLY TMA: THEORY AND LIMITATION.}

Consider the source-observer encounter depicted in figure 1. The source located at the coordinates $\left(r_{x s}, r_{y s}\right)$ moves with a constant velocity $\left(v_{x s}, v_{y}\right)$. The state vectors of the source and the observer are [3]:

$$
X_{s} \triangleq\left[r_{x s}, r_{y s}, v_{x s}, v_{y s}\right]^{t} \quad X_{o} \triangleq\left[r_{x o}, r_{y o}, v_{x o}, v_{y o}\right]^{t} .
$$

\footnotetext{
${ }^{1}$ This work bas been oupported by DCN Ingenierie-Sud France.
}

In terms of relative state vector $X$, defined by $X=$ $X_{\mathrm{s}}-X_{0} \triangleq\left[r_{x}, r_{y}, v_{x}, v_{y}\right]^{t}$, the discrete time equation takes the following form (uniform rectilinear motion):

$$
X\left(t_{k}\right)=\Phi\left(t_{k}, t_{k-1}\right) X\left(t_{k-1}\right)+U\left(t_{k}\right)
$$

where:

$\Phi\left(t_{k}, t_{k-1}\right)=\left(\begin{array}{cc}\operatorname{Id} & \left.\left(t_{k}-t_{k-1}\right)\right) \operatorname{Id} \\ 0 & \text { Id }\end{array}\right), \quad$ Id $\triangleq\left(\begin{array}{ll}1 & 0 \\ 0 & 1\end{array}\right)$.

and $U\left(t_{k}\right)$ accounts for the effects of the observer accelerations.

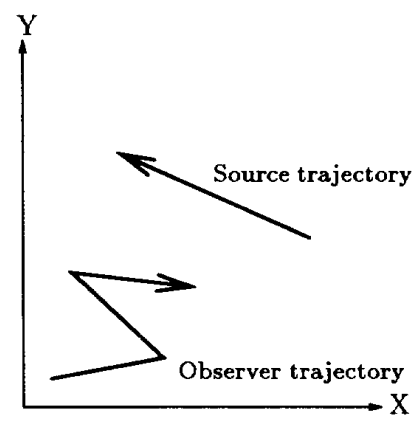

Figure 1: Source-Observer encounter.

Classicaly, the available measurements are the estimated angles $\theta_{t}$ (bearings) from the observer's platform to the source or, equivalently, the estimated value of the spatial frequencies $k_{\mathrm{t}}\left(k_{t}=\sin \theta_{t} / \lambda, \lambda\right.$ : wavelength), so that:

$$
\hat{k}_{\mathrm{t}}=k_{\mathrm{t}}+\nu_{\mathrm{t}}
$$

where $\nu_{t}$ is an additive white gaussian noise with a variance $\sigma_{\nu}^{2}$ given by the classical Woodward's formula, in the case of a linear array regularly sampled in space and for a unique source in the array broadside:

$$
\sigma_{\nu}^{2}=\frac{3(1+p \rho)(2 N-1)}{p^{2} \rho^{2}\left(p^{2}-1\right) \pi^{2} d^{2} N(N+1)}
$$

with:

signal to noise ratio number of sensors number of snapshots intersensor distance 
This type of model is based on the assumption of a rectilinear motion of the source. This may be a crucial limitation when the source changes its velocity. In fact interesting sources are those which maneuver for tactical reason, so our attention must be focused on them. There exists algorithms to track maneuvering targets, but generally they got poor results for TMA applications because of their approach of the problem. Some of them allow certain types of maneuver, and choose among them thanks to a likelihood ratio. Few others increase the dimension of the state vector (adding the acceleration) when the innovation is too important. None of them works correctly for real scenarios, wether the maneuver is not provided in the algorithm or the modification of the velocity is not adequate.

That's why a probabilistic model of the source's motion must be considered and more precisely a Markov model.

\section{HIDDEN MARKOV MODEL.}

The advantage of the Markov model $[4,6]$ over the other approaches is that one do not have to define different types of maneuvers with the risk that the source doesn't follow any of them. One has just to assume that a source won't change its velocity radically between two instants.

The targets are characterized by their position $\left(x_{t}, y_{t}\right)$ and their velocity $\left(v_{x t}, v_{y t}\right)$ in cartesian coordinates. These variables can take any value in a finite denombrable set. A part of the 2D position space and velocity space are taken and sampled regularly or not.

In order to define the state transition matrix, direct probability calculations give:

$$
\begin{aligned}
E_{t} & =\left[x_{t}, y_{t}, v_{x t}, v_{y t}\right]=\left[X_{t}, V_{t}\right] \\
\operatorname{Pr}\left(E_{t+1} \mid E_{t}\right) & =\operatorname{Pr}\left(X_{t+1}, V_{t+1} \mid X_{t}, V_{t}\right) \\
& =\operatorname{Pr}\left(X_{t+1} \mid X_{t}, V_{t}\right) \operatorname{Pr}\left(V_{t+1} \mid X_{t+1}, X_{t}, V_{t}\right) .
\end{aligned}
$$

If, besides this, the transition over the velocities is supposed independent from the transition over the positions, one can define a transition on the position space (centered on the estimated position) and a transition on the velocity space. All the possibilities are described on figure 2 for the position transitions and on figure 3 for the transitions in the velocity space.

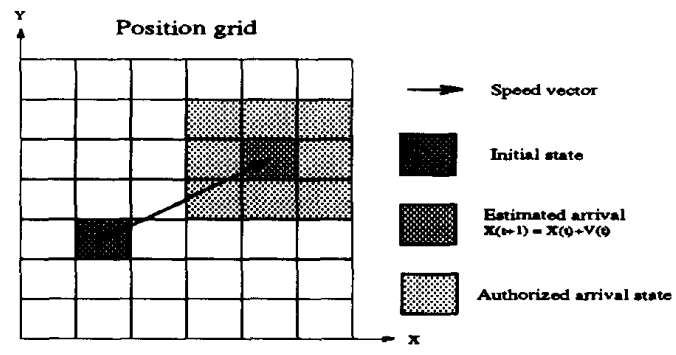

Figure 2: Position transitions.

The conventional beamforming gives the energy density on all directions, so it can be used to compute the observation which is proportional to the probability for each cell to contain a source.

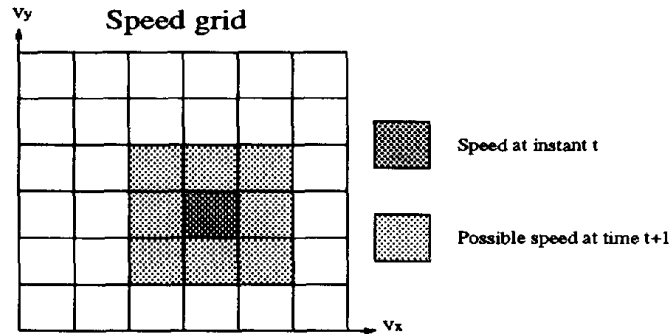

Figure 3: Speed transitions.

In figure 4 there is the result of the process via the Viterbi algorithm of a source evolving along a circular path. The source executes a U-turn, classical trackers would fail but the Viterbi algorithm, is able to track and localize it.
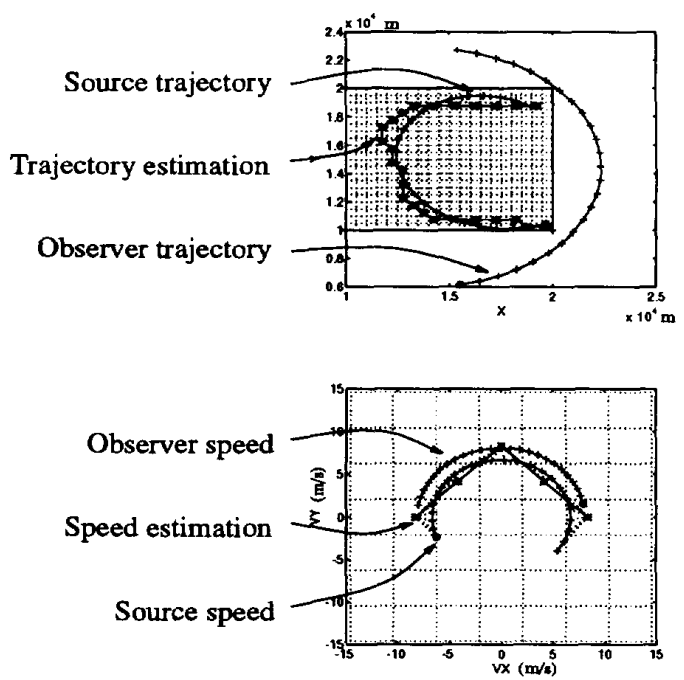

Figure 4: Source along a circular path.

\section{OPTIMAL TRANSITION MATRIX.}

First of all one can try to find the transition matrices which satisfy a criterion over a set of trajectories. In order to simplify the computation, our main hypothesis is that the innovation is gaussian:

$$
\begin{aligned}
V_{t+1}-V_{t} & \equiv \mathcal{N}\left(0, \sigma_{v} \operatorname{Id}_{2}\right) \\
X_{t+1}-\left(X_{t}+\delta T V_{t}\right) & \equiv \mathcal{N}\left(0, \sigma_{x} \operatorname{Id}_{2}\right)
\end{aligned}
$$

Given $\sigma_{x}$ and $\sigma_{v}$ one can compute the corresponding transition matrices. To compute the transition probability one integrate the density function on the state square and normalizes. The covariance matrices have the form cId so the surface integrals become the product of two integrals, transition matrices become dyadic product $D_{x} D_{y}^{t}$ with $D_{x}$ 
and $D_{y}$ centrosymmetric vectors (symmetry of the gauss function). For example a 3 by 3 transition matrix will be given by:

$$
\begin{aligned}
D_{s}= & {\left[b_{s} a_{s} b_{s}\right]^{t} } \\
T(s)= & D_{s} D_{s}^{t}=\left[\begin{array}{ccc}
b_{s}^{2} & a_{s} b_{s} & b_{s}^{2} \\
a_{s} b_{s} & a_{s}^{2} & a_{s} b_{s} \\
b_{s}^{2} & a_{s} b_{s} & b_{s}^{2}
\end{array}\right] \\
\text { with : } & a_{s}=\operatorname{erf}(s) / \operatorname{erf}(3 s) \\
& b_{s}=(\operatorname{erf}(3 s)-\operatorname{erf}(s)) / 2 \operatorname{erf}(3 s)
\end{aligned}
$$

$s$ is related to $\sigma$ and to the size of the square which represents the state $(s=s i z e / 2 \sigma)$, and $\operatorname{erf}(x)$ is the standard error function.

In order to find the optimal matrices, multiple trajectories are generated from a starting point, an initial heading, a speed (constant in modulus) and a curvature. These trajectories are translated into a series of transitions (we have to set the size of the state-square) that are counted. After the normalization one obtain the optimal matrix $(T)$ for the set of trajectories that have been generated. One look for the normalized dyadic matrix $(T(s))$ so that:

$$
s^{*}=\arg \min _{s} \sum_{i, j}\left(T_{i j}(s)-T_{i j}\right)^{2}
$$

This is done by looking for the zero of the derivative of the square error function. Once $s$ is obtained, $\sigma$ is deduced and one can compute the transition matrix for a different size of state.

The algorithm has been conducted for the following set of trajectories:

- 10 different starting point randomly chosen.

- 10 speeds regularly spaced between 0 and $30 \mathrm{~m} / \mathrm{s}$.

- 20 initial headings (random).

- 31 different curvature linearly spaced between $-10^{-3}$ and $10^{-3}$

The algorithm found a zero of the derivative for:

$$
\begin{aligned}
& \sigma_{x}=560 \\
& \sigma_{v}=4.37
\end{aligned}
$$

These variances induce a vector which is the eigen vector corresponding to the highest eigen value of the optimal transition matrix.

\section{OPTIMAL CONTROL.}

In order to estimate the trajectory of the source the observer has to maneuver. Different maneuvers will yield different quality of trajectory estimation. This section deals with the optimal maneuver of the observer platform when the observations consist of the exact state of the source and for bearings-only source tracking [1].

\subsection{EXACT STATE OBSERVATIONS.}

The state $(\mathcal{S})$ and control $(\mathcal{C})$ space are finite. The state of the markov chain consist of the relative position and velocity vector between the source and the observer: $X_{k}=$ $\left(r_{x}(k), r_{y}(k), v_{x}(k), v_{y}(k)\right)^{t}$. The control space is the set of all possible evolutions of the position and velocity of the observer: $U_{k}=X_{o}(k+1)-X_{o}(k)$. The transition matrix $\left(\left\{P_{i j}(d)\right\}_{i, j \in S} d \in \mathcal{C}\right)$ depends on the control state (firgure 5) and the cost of source transition which depends also on the relative position and velocity between the source and the observer and thus is a function of the control $\left(\left\{C_{i, j}(d)\right\}_{i, j \in S} d \in\right.$ $\mathcal{C})$.

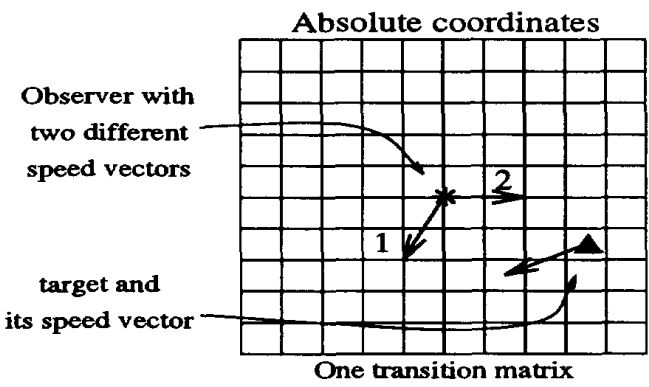

whatever the observer motion

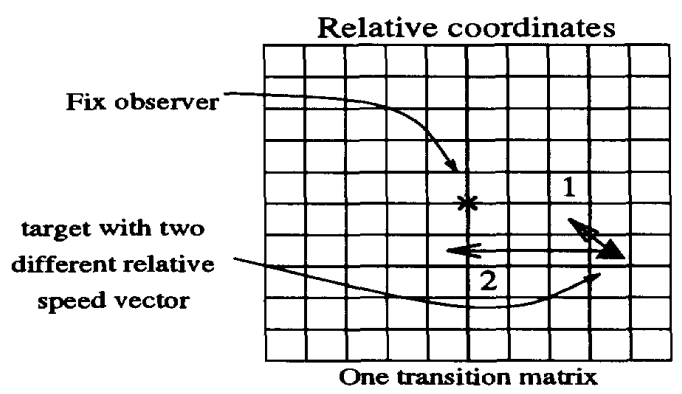

for each observer motion

Figure 5: Transition matrices and control.

This cost function has to be relevant of the estimability of the target trajectory. One can choose the inverse of the determinant of the Fisher information matrix computed on the data received between $t=k \delta T$ and $t=(k+1) \delta T$. At the initial instant $k=0$ the system is in a given initial state $X_{0}$. If a decision $U_{0}$ is taken at that time-instant the state is transferred to $X_{1}$ according to the transition probability $P_{X_{0}, X_{1}}\left(U_{0}\right)$. Associated with this transition probability we have the cost $C_{X_{0}, X_{1}}\left(U_{0}\right)$. At instant $k=1$ the process is transferred to $X_{2}$ while a new decision is taken $U_{1}$ and so on. The problem is to find the control policy $\eta=\left(\eta_{0}, \cdots, \eta_{N-1}\right)$ such that $U_{k}=\eta_{k}\left(X_{k}, U_{k-1}\right) k=0, \cdots, N-1$ so that:

$$
J(U)=\mathbf{E}\left\{\sum_{k=0}^{N-1} C_{X_{k}, X_{k+1}}\left(U_{k}\right)\right\}
$$

is minimized. 
The dynamic programming equation is:

$$
\begin{gathered}
J^{*}(k, i)=\min _{d \in \mathcal{C}} \sum_{j \in \mathcal{S}}\left[C_{i j}(d)+J^{*}(k+1, j)\right] P_{i j}(d) \\
\quad i \in \mathcal{S}, \quad k=N-1, \cdots, 0
\end{gathered}
$$

The value of the control which gives the minimum is the optimal decision rule at time $k$ if one is in state $i$ at that moment. One has to solve this equation backward in time with the terminal condition:

$$
J^{*}(N, i)=0, \quad i \in \mathcal{S}
$$

\subsection{PARTIAL OBSERVATIONS (BEARINGS-ONLY).}

The state and the control space are identical as in the previous subsection, but the exact state can no longer be observed. In fact one can measure the bearing between the south-north axis and the source-observer direction:

$$
Y_{k}=h\left(X_{k}\right)+V_{k}, \quad V_{k} \equiv \mathcal{N}\left(0, \sigma^{2}\right)
$$

The transition and cost matrices still depend on the control and one has the same cost function to minimize. As the exact state of the markov chain cannot be measured, one can construct the vector of all informations available at time $k$ :

$$
Z^{k} \triangleq\left(Y^{k}, U^{k-1}\right)=\left(Z^{k-1}, Y_{k}, U_{k-1}\right)
$$

The dynamic programming equation takes the form:

$$
\begin{aligned}
J^{*}\left(k, z^{k}\right)= & \min _{d \in \mathcal{C}}\left[\mathbf{E}\left\{C_{X_{k}, X_{k+1}}(d) \mid Z^{k}=z^{k}\right\}\right. \\
& \left.+\mathbf{E}\left\{J^{*}\left(k+1, Z^{k+1}\right) \mid Z^{k}=z^{k}, U_{k}=d\right\}\right]
\end{aligned}
$$

with the terminal condition:

$$
J^{*}\left(N, z^{N}\right)=0
$$

These equations are very difficult to solve, so one should try to find a simplification of this problem via a sufficient statistics. The information state vector must be replaced by a much smaller dimensionality vector which will enable the computation. Define [1]:

$$
\begin{aligned}
\pi_{k \mid l}\left(i \mid z^{l}\right) & \triangleq \operatorname{Pr}\left(\left\{X_{k}=i\right\} \mid z^{l}\right) \\
\Pi_{k \mid l} & =\left(\pi_{k \mid l}\left(i \mid z^{l}\right)\right)_{i \in \mathcal{S}}
\end{aligned}
$$

Some calculation yields the iterative equation:

$$
\Pi_{k+1 \mid k+1}=\frac{\Pi_{k \mid k}\left(z^{k}\right) P\left(U_{k}\right) D\left(Y_{k+1}\right)}{\Pi_{k \mid k}\left(z^{k}\right) P\left(U_{k}\right) D\left(Y_{k+1}\right) \underline{1}}
$$

with the initial condition:

$$
\Pi 0 \mid 0\left(z^{0}\right)=\frac{\Pi_{0} D\left(Y_{0}\right)}{\Pi_{0} D\left(Y_{0}\right) \underline{1}}
$$

where:

$$
\begin{aligned}
& \underline{1}=(1,1, \cdots, 1)^{t} \\
& D\left(Y_{k+1}\right)=\operatorname{diag}\left\{\exp \left(Y_{k+1} h(i) / \sigma^{2}\right) \exp \left(-h(i)^{2} / 2 \sigma^{2}\right)\right\}_{i \in S}
\end{aligned}
$$

$$
\begin{aligned}
J^{s}(k, \pi) & =\min _{d \in \mathcal{C}}\left[\mathbf{E}\left\{C_{X_{k} X_{k+1}}\left(U_{k}\right) \mid \Pi_{k \mid k}=\pi\right\}\right. \\
& =\mathbf{E}\left\{J ^ { s } \left(k+1, \Pi_{k+1 \mid k+1}\left(Z^{k+1} \mid \Pi_{k \mid k}\left(Z^{k}=\pi, U_{k}=d\right)\right\} .\right.\right.
\end{aligned}
$$

with the initial condition

$$
J^{s}(N, \pi)=0
$$

\section{CONCLUSION.}

After a brief review on bearings-only target tracking, hidden markov model has been introduced as an answer to maneuvering target tracking. The gaussian assumption for the innovation, enabled the computation of the covariance matrices (position and velocity) associated with the transition matrix. Finally, the problem of observer's trajectory optimization has been considered via the estimation and control of partially observed markov chain. Simulation results show that this type of probabilistic model for the source motion is adapted to highly and slowly maneuvering target tracking.

\section{REFERENCES}

[1] A. Bagchi. Optimal control of stochastic systems. Prentice-Hall, 1993.

[2] P.R. Kumar and Pravin Varaiya. Stochastic systems (estimation, identification and adaptive control). PrenticeHall, 1986.

[3] Steven C. Nardone, Allen G. Lindgren, and Kai F. Gong. Fundamental properties and performance of conventional bearings-only target motion analysis. IEEE Transactions on automatic control, 29(9):775-787, sep 1984.

[4] L. R. Rabiner and B. H. Juang. An introduction to hidden markov models. IEEE Transactions on acoustics, speech and signal processing, 4, jan 1986.

[5] Roy L. Streit. The moments of matched and mismatched hidden markov models. IEEE Transactions on acoustics, speech and signal processing., 38(4):610-622, April 1990.

[6] Xianya Xie and Robin J. Evans. Multiple target tracking and multiple frequency line tracking using hidden markov model. IEEE Transactions on signal processing, 39(12):2659-2676, December 1991. 